\title{
Two-dimensional fluorescence spectroscopy: A novel approach for controlling fed-batch cultivations
}

\author{
K. Hantelmann, M. Kollecker, D. Hüll, B. Hitzmann, T. Scheper* \\ Institut für Technische Chemie, Callinstraße 3, 30167 Hannover, Germany
}

Received 22 April 2005; received in revised form 27 June 2005; accepted 15 July 2005

\begin{abstract}
In industrial fed-batch cultivations it is often necessary to control substrate concentrations at a low level to prevent the production of overflow metabolites and thus optimize the biomass yield. A new method for on-line monitoring and fed-batch control based on fluorescence measurements has been developed. Via instantaneous in situ measurements and multivariate data analysis a chemometric model has been established, which enables the rapid detection of ethanol production at aerobic Saccharomyces cerevisiae fed-batch cultivations. The glucose feed rate is controlled by predicting the metabolic state directly from the fluorescence intensities. Thus, ethanol production could be avoided completely while increasing the biomass yield accordingly. The robust instrumentation is suitable for industrial applications.
\end{abstract}

(C) 2005 Elsevier B.V. All rights reserved.

Keywords: Fed-batch; Fluorescence; On-line monitoring; Ethanol inhibition; S. cerevisiae

\section{Introduction}

Since aerobic fed-batch cultivations are commonly applied for the production of Saccharomyces cerevisiae as baker's yeast as well as for the production of recombinant proteins, it has always been an economic matter of interest to optimize the process operation. Due to the Crabtree effect (De Dekken, 1966) S. cerevisiae produces ethanol even under fully aerobic conditions, which is believed to be caused by a limited respira-

\footnotetext{
* Corresponding author. Tel.: +49 511762 2509; fax: +495117623004.

E-mail address: Scheper@iftc.uni-hannover.de (T. Scheper).
}

tory capacity (Sonnleitner and Käppeli, 1986). Above a critical glucose concentration, which depends on the strain, media composition and the oxygen supply, glucose is consumed oxidoreductively (Van Hoek et al., 1999). In order to maintain oxidative growth, several approaches have been published to control the feed rate at a level below the critical value, beyond which ethanol is produced and therefore the biomass yield decreased. Mostly, the feed rate is controlled via on-line measurements or estimations of either glucose (Arndt and Hitzmann, 2004; Chen et al., 2003; Shimizu et al., 1988) or ethanol (Dairaku et al., 1982; Valentinotti et al., 2003). Recently, there have also been attempts to monitor and control fed-batch cultivations 
via exit-gas analysis and calculating the metabolic state based on black box reaction equations (Claes and Van Impe, 2000; Jobé et al., 2003).

Though almost all approaches are suitable for online measurements, there's still the problem of a certain time delay. And so far no in situ method has been presented, which delivers instantaneous results. As to the possibility of in situ measurements optical sensors have long been applied for non-invasive process monitoring (Ulber et al., 2003; Stärk et al., 2002). For monitoring fed-batch cultivations of yeasts, and especially the formation of ethanol, studies report the use of MIR spectroscopy (Mazarevica et al., 2004) and Raman spectroscopy (Gomy et al., 1988; Shaw et al., 1999) for example. Nevertheless, those methods have not yet been applied to control the feed rate but merely to predict concentrations of glucose and ethanol.

The present work presents an in situ method suitable for the rapid detection of ethanol production via two-dimensional (2D) fluorescence spectroscopy. The concept is based on the detection of changes in the concentration of mainly NADH and flavins (FAD, FMN) occurring when the metabolism changes from oxidative to oxidoreductive. Those changes are monitored in a first fed-batch cultivation, evaluated chemometrically and finally used to control the feed rate. Prior to the application a sensitivity analysis was carried out to evaluate the information contained in the spectra depending on the resolution.

\section{Materials and methods}

\subsection{Fluorescence sensor}

The in situ fluorescence measurements are carried out using the BioView ${ }^{\circledR}$ sensor (Delta Light and Optics, Denmark), a robust instrument designed for industrial applications (Marose et al., 1998; Scheper et al., 1999). The system is based on multichannel fluorescence detection. It uses two independently rotating filter wheels with 16 different filters each, for excitation (270-550 $\mathrm{nm})$ and emission (310-590 nm). Those filters can be chosen individually according to the process requirements. A version using up to 20 individual filters was additionally used in the experiments. Thus, the two-dimensional landscape of the measured matrix
Table 1

Concentration of fluorophors used for the sensitivity analysis

\begin{tabular}{|c|c|c|}
\hline Fluorophor & $\begin{array}{l}\text { Starting } \\
\text { concentration } \\
\left(\mu \mathrm{g} \mathrm{mL}^{-1}\right)\end{array}$ & $\begin{array}{l}\text { Fluorescence } \\
\text { maximum }\end{array}$ \\
\hline Rhodamine $\mathrm{B}^{\mathrm{a}, \mathrm{b}}$ & $2.5 \times 10^{-4}$ & $\overline{E x 560 / e m 575}$ \\
\hline Alexa Fluor $430^{\mathrm{a}, \mathrm{b}}$ & $2.5 \times 10^{-3}$ & Ex430/em520 \\
\hline $\begin{array}{l}\text { 4-Methylumbelliferryl- } \\
\text { phosphate }^{\mathrm{a}, \mathrm{b}}\end{array}$ & $5 \times 10^{-4}$ & Ex320/em380 \\
\hline FITC $^{\mathrm{a}, \mathrm{b}}$ & $1.25 \times 10^{-4}$ & Ex500/em520 \\
\hline Propidium iodide $\mathrm{e}^{\mathrm{a}, \mathrm{c}}$ & $5 \times 10^{-3}$ & Ex540/em590 \\
\hline Hoechst $33342^{\mathrm{a}, \mathrm{c}}$ & 0.1 & Ex355/em465 \\
\hline Sytox Green ${ }^{\mathrm{a}, \mathrm{c}}$ & 60 & Ex505/em520 \\
\hline Bodipy & 0.04 & Ex490/em500 \\
\hline
\end{tabular}

Fluorescence maxima and composition of mixtures.

${ }^{\text {a }}$ Contained in mixture 1.

${ }^{b}$ Contained in mixture 2.

${ }^{\mathrm{c}}$ Contained in mixture 3.

allows for the simultaneous detection of various fluorophors.

The recording of a whole spectrum takes $90 \mathrm{~s}$ with a resolution of $20 \mathrm{~nm}$, whereas the time can be reduced by reducing the number of filters, e.g. if single spectral regions contain no information. The BioView ${ }^{\circledR}$ sensor is placed in a high-grade steel box for protection against harsh environments (e.g. high temperature, moisture) and electromagnetical interferences. For the in situ measurements it is connected by a bifurcated liquid light conductor (Lumatec, Germany) to a $25 \mathrm{~mm}$ standard port of the bioreactor, which contains an adapter with a quartz glass window to ensure the non-invasive monitoring and to eliminate the risk of contamination. A single-fiber asynchronous modem is used for data transfer.

\subsection{Sensitivity analysis}

The sensitivity analysis was carried out using a Hitachi F-4500 (Hitachi, Japan).

Eight fluorophors were measured first individually and afterwards in three different mixtures to obtain 2D fluorescence spectra covering the whole range from 270 to $590 \mathrm{~nm}$ for excitation and $310-360 \mathrm{~nm}$ for emission (see Table 1). For each sample three different concentrations were measured in wavelength steps of $5 \mathrm{~nm}$. Therefore, the starting solutions were diluted 1:1 and 1:4, respectively, with PBS buffer at pH 7. 


\subsection{Strain and media}

The $S$. cerevisiae wildtype strain H620 was grown on Schatzmann medium (Schatzmann, 1975), which was supplemented with $5.8 \mathrm{~g} \mathrm{~L}^{-1}$ sodium citrate for the preculture. The fed-batch cultivations were started after an initial batch cultivation on $15 \mathrm{~g} \mathrm{~L}^{-1}$ glucose. The feed contained $50 \mathrm{~g} \mathrm{~L}^{-1}$ glucose.

\subsection{Culture conditions and bioprocess set-up}

The cultivations were carried out in a $2 \mathrm{~L}$ bioreactor. The temperature was controlled at $30^{\circ} \mathrm{C}$, the $\mathrm{pH}$ at 5.5 using $\mathrm{NaOH}(10 \%)$ and $\mathrm{HCl}(4 \mathrm{M})$. The stirrer speed was $1200 \mathrm{rpm}$ with an air inlet flow of $5 \mathrm{~L} \mathrm{~min}^{-1}$.

Exit gas (EGAS 2, Hartmann \& Braun, Germany), dissolved oxygen (Inpro 6000, Mettler-Toledo, USA), and $\mathrm{pH}$ (FermProbe T635, Broadley \& James, USA) were measured on-line. Glucose measurements were carried out with a flow injection analysis (FIA) system (Anasyscon, Germany) during the first fed-batch cultivation (Hitzmann et al., 2000). In this case, the feed rate was controlled by glucose concentration set-points.

Fluorescence measurements were taken continuously, i.e. every 90 s. For the fluorescence based fedbatch control the measuring PC of the BioView ${ }^{\circledR}$ sensor was connected to a second PC controlling the feed pump (ACCU CP10, SciLog, USA).

\subsection{Off-line analysis}

For off-line analysis samples were taken every $30 \mathrm{~min}$. Biomass (dry weight) was determined gravimetrically after centrifugation in $2 \mathrm{~mL}$ Eppendorf reaction vessels. Ethanol was determined by gas chromatography (GC 14B, Shimadzu, Japan), off-line glucose concentrations reflectometrically (Reflectoquant ${ }^{\circledR}$, Merck, Germany).

\section{Results and discussion}

\subsection{Sensitivity analysis of $2 D$ fluorescence spectra}

Duration and precision of measurements are often contrary when monitoring processes on-line. As to optical sensors, given a spectral range the recording time of a spectrum depends on the resolution for exam-
Table 2

Relative slope error of the linear regression calculated from the scores of the reduced spectra vs. the original spectra

\begin{tabular}{ll}
\hline Resolution & Relative error of slope $(\%)$ \\
\hline 5 & 0 \\
10 & 0.13 \\
15 & 1.06 \\
20 & 1.85 \\
25 & 2.54 \\
30 & 4.49 \\
\hline
\end{tabular}

ple. To evaluate the loss of information with decreased resolution a sensitivity analysis was carried out. For that purpose 33 spectra of different fluorophors and mixtures were recorded with a resolution of $5 \mathrm{~nm}$ (see Section 2.2). Afterwards, reduced spectra with resolutions of $10,15,20,25$ and $30 \mathrm{~nm}$ were generated from each original spectrum by leaving out the corresponding intensity readings. To analyze as well as quantify the content of information a principal component analysis was carried out individually for each original and reduced spectrum (with 30 calculated principal components (PC) and full cross validation). Plotting the first scores of each reduced spectra set versus the first score of the original spectra (resolution $\Delta \lambda=5 \mathrm{~nm}$ ) gives the result as shown in Fig. 1.

The linear regression points out that the slope decreases with a decreasing resolution while the distance from the first bisecting line $(5 \mathrm{~nm}$ versus $5 \mathrm{~nm}$ ) increases. This already indicates that the sensitivity lessens with a decreasing resolution. Furthermore, the relative error of the slope increases up to $4.5 \%$ with a resolution of $30 \mathrm{~nm}$ (see Table 2).

With regard to the measurement time and sensitivity a resolution of $20 \mathrm{~nm}$ is a good compromise and is used for the following experiments accordingly.

\subsection{Monitoring the oxidative-oxidoreductive transition}

With S. cerevisiae, the change of oxidative to oxidoreductive metabolism is directly correlated to a change in the concentrations of biogenic fluorophors. If the metabolism is purely oxidative, one observes a steady increase in the concentrations of the electron carriers NADH and the coenzymes FAD and FMN due to the cell growth. Accordingly, a steady increase in the fluorescence intensities is detected. 


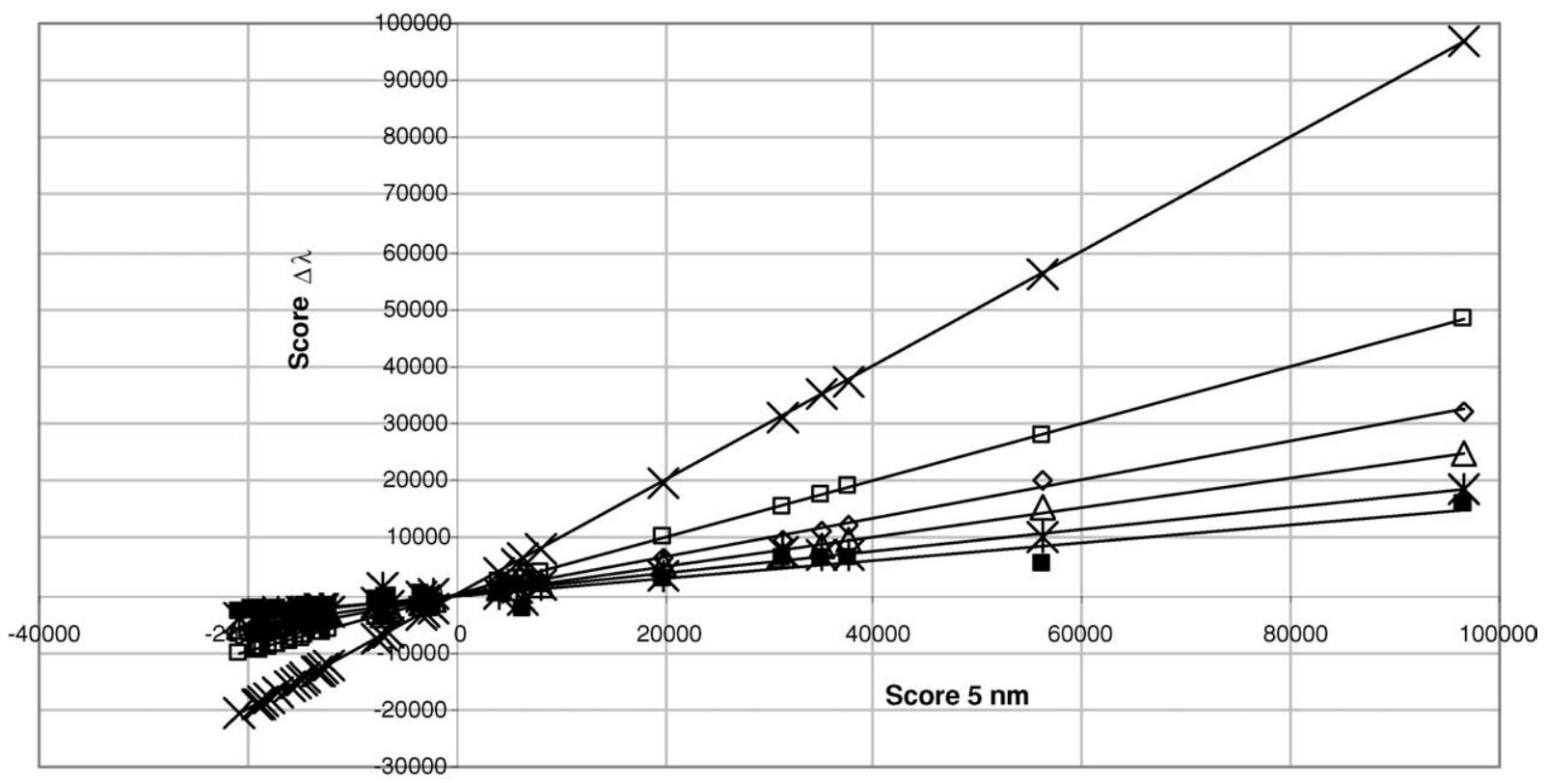

Fig. 1. Scores of the first PC of the spectra with $\Delta \lambda=5(\times), 10(\square), 15(\diamond), 20(\Delta), 25(\mathcal{X})$ and $30 \mathrm{~nm}(\boldsymbol{\square})$ vs. the scores of the first PC of the original spectra $(\Delta \lambda=5 \mathrm{~nm})$ with linear regression line.

For the production of ethanol additional NADH is needed for electron transport to acetaldehyde, which is thus reduced to ethanol. Therefore, an accumulation of reduced redox equivalents $\left(\mathrm{NADH}, \mathrm{FADH}_{2}\right.$ and $\mathrm{FMNH}_{2}$ ) occurs shortly before the oxidoreductive metabolism sets in. Additionally, the concentration of the fluorescent oxidized forms FAD and FMN decreases. These significant changes are detected via fluorescence measurements and serve as the signal for the beginning ethanol production (Schügerl et al., 1993).

The critical glucose concentration, above which the Crabtree effect sets in, is about $0.04 \mathrm{~g} \mathrm{~L}^{-1}$, depending on the experimental conditions (Pham et al., 1998). Under the present conditions it has been determined to be $0.05 \mathrm{~g} \mathrm{~L}^{-1}$ glucose.

To monitor the metabolic changes, a fed-batch cultivation with FIA based glucose control was run. By fixing different set-points, metabolic changes were forced and detected by fluorescence measurements. Fig. 2 shows the feed profile as well as the measured variables during the cultivation.

The glucose set-point was increased five times above the critical concentration of $0.05 \mathrm{~g} \mathrm{~L}^{-1}$. The FIA measurements exhibit a time delay of 4 min, which is why the set point concentration is not always reached exactly and in time. Nevertheless the system reacts to the actual glucose concentration. While the glucose concentration is low, no significant ethanol production is detected. As soon as the concentration is raised, oxidoreductive metabolism starts. The change is observable in the course of the $\mathrm{CO}_{2}$ production as well. The respiratory quotient (RQ) distinctly surpasses 1 at those times (data not shown). The course of the fluorescence intensities shows the described abrupt increase as to $\mathrm{NADH}$ and decrease as to the flavins, respectively. The information contained in the spectra was further used to build a model that is suitable for a fluorescence based glucose control.

\subsection{Chemometric model for the fluorescence based glucose control}

First, to correlate the fluorescence data and the ethanol production, an ethanol production value (EP value) was defined, which is 0 (no ethanol production) or 1 (ethanol production), respectively. Thus, every spectrum from the fed-batch cultivation described 

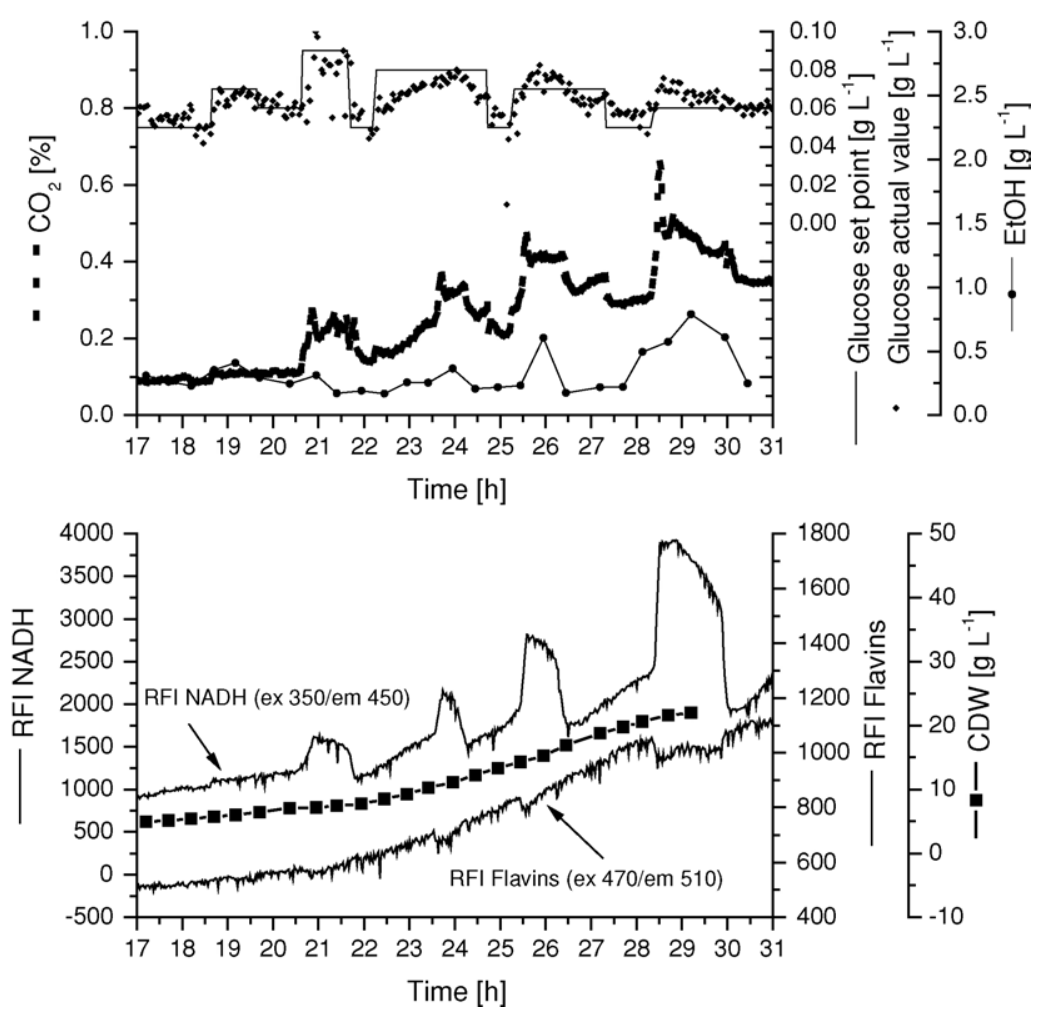

Fig. 2. S. cerevisiae fed-batch cultivation with FIA based glucose control. Time course of the carbon dioxide production, glucose and ethanol concentration, cellular dry weight (CDW) and of the relative fluorescence intensities (RFI) of NADH and flavins at different glucose set-points.

above was classed with an EP value. In total 1059 spectra were used for the chemometric model calculation. Subsequently, a PLS regression model was built, which is able to predict the EP values from the spectra (Software The Unscrambler ${ }^{\circledR}$, CAMO ASA, Norway). Here three latent variables (factors) were found to be adequate. To eliminate the influence of different cultivation conditions on the fluorescence intensities and ensure the transferability of the model, the time alteration of the EP value was determined for the process control. It was calculated according to Eq. (1) as the derivative with respect to time of the EP value using a Savitzky-Golay filter:

$$
\begin{aligned}
\mathrm{EP}_{n}^{\prime}= & \frac{-2 \mathrm{EP}_{n-4}-\mathrm{EP}_{n-3}+\mathrm{EP}_{n-1}+2 \mathrm{EP}_{n}}{10 \Delta t} \\
+ & \frac{4 \mathrm{EP}_{n-4}-2 \mathrm{EP}_{n-3}-4 \mathrm{EP}_{n-2}}{7 \Delta t}
\end{aligned}
$$

$n$ is the index of the measurement and $\Delta t$ the measuring interval of the BioView ${ }^{\circledR}$ sensor. Finally, the noise was reduced by calculating the metabolic (MB) value as follows:

$$
\mathrm{MB}_{n}=\mathrm{EP}_{n}^{\prime} A+\mathrm{EP}_{n-1}^{\prime}(1-A) \quad A=0.25
$$

Thus, by calculating the MB value from the spectra, it is possible to predict the metabolic state at any point of time during a cultivation. The limiting value, above which oxidoreductive metabolism takes place, is 1 . Fig. 3 shows the calculated MB values for the described fed-batch cultivation (Fig. 2).

To control the feed pump, a program written in Borland Pascal 7.0 was employed, which is based on a simple state model for exponential growth. The cellular dry weight, the glucose concentration and the reactor volume serve as state variables. The feed rate $\dot{V}_{\text {Feed }}(t)$ based on that model is calculated using the following 


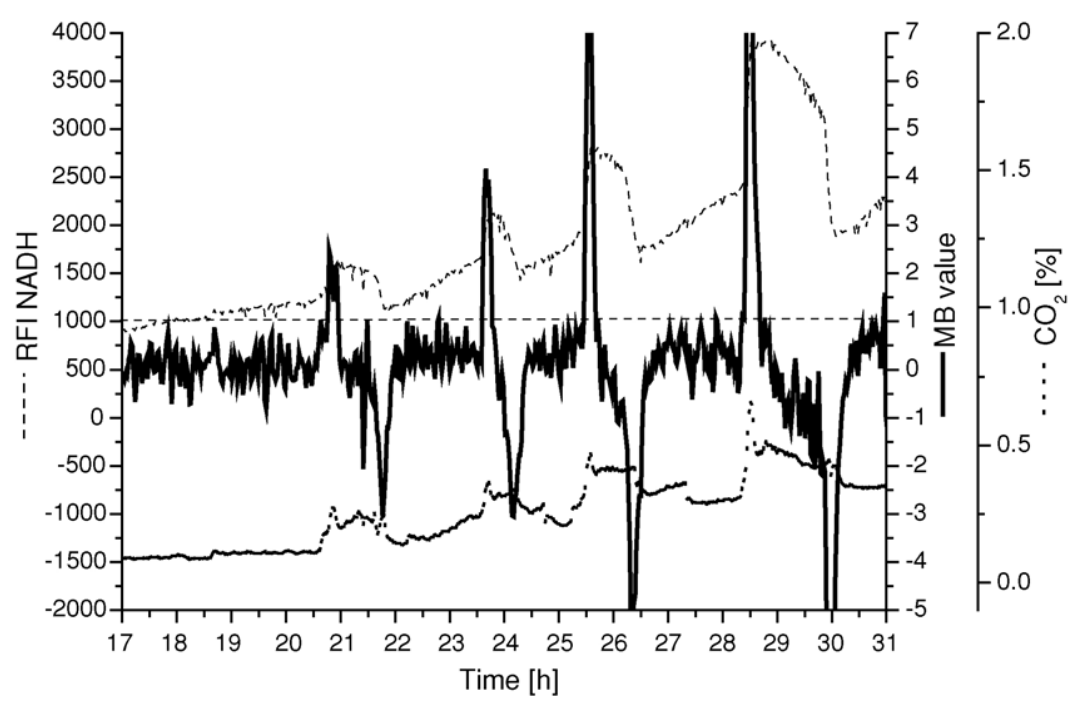

Fig. 3. Calculated metabolic values for each point of time during the S. cerevisiae fed-batch cultivation with FIA based glucose control. Time course of carbon dioxide production and NADH fluorescence intensity.

equation:

$\dot{V}_{\text {Feed }}(t)=\frac{\mu X(t) V(t)}{Y\left(S_{0}-S(t)\right)}$

In this equation, $\mu$ is the specific growth rate for the model, $X(t)$ the cellular dry weight, $V(t)$ the reactor volume, $Y$ the yield coefficient $\left(Y=0.5 \mathrm{~g} \mathrm{~g}^{-1}\right), S_{0}$ the glucose concentration in the feed, $S(t)$ the glucose concentration and $t$ is the time.

The specific growth rate in the model is chosen slightly higher than the real specific growth rate of the cells. Hence, the glucose concentration in the broth increases steadily until a threshold concentration is reached and oxidoreductive metabolism begins. By continuously determining the MB value from the recorded fluorescence spectra, this change is detected in-time and the feed rate is adjusted accordingly.

\subsection{Fluorescence based glucose control}

The described model has been employed to control another S. cerevisiae fed-batch cultivation (Fig. 4). The chemometric model was not modified for the application. No base line adjustment or other modifications were necessary, because the change of the EP value with respect to time (the MP value) was used as indicator. This is independent of absolute intensity values of the fluorescence. The cultivation was run over $11 \mathrm{~h}$, during which $2 \mathrm{D}$-fluorescence spectra were recorded every $90 \mathrm{~s}$. From every spectrum the according MB value was calculated immediately. The critical value, above which ethanol production takes place, is 1 . During the process five control operations were carried out automatically, for a MB value greater than 1 was calculated (marked by arrows in Fig. 4).

Although the critical glucose concentration was reached several times, the production of ethanol could be inhibited completely due to the in-time reduction of the feed rate. During the process $63 \mathrm{~g}$ glucose were fed. The biomass increased from 4.5 to $15.9 \mathrm{~g} \mathrm{~L}^{-1}$. The biomass yield was $0.58 \mathrm{gg}^{-1}$, which is a definite sign, that pure oxidative growth took place. The yield at oxidoreductive growth lies significantly lower at about $0.3 \mathrm{~g} \mathrm{~g}^{-1}$ (Woehrer and Roehr, 1981). In calculating the overall yield, the loss of biomass due to the off-line samples was taken into account. The glucose concentration was constantly close to the critical concentration of $0.4-0.5 \mathrm{~g} \mathrm{~L}^{-1}$. This signifies that the glucose fed to the cells was metabolized at once and completely. With every control operation, because of the reduced feed rate, a short-term decrease in the glucose concentration could be detected. The change of metabolism was clearly observable in the time course of the fluorescence intensity of $\mathrm{NADH}$ and the $\mathrm{CO}_{2}$ production as well. 

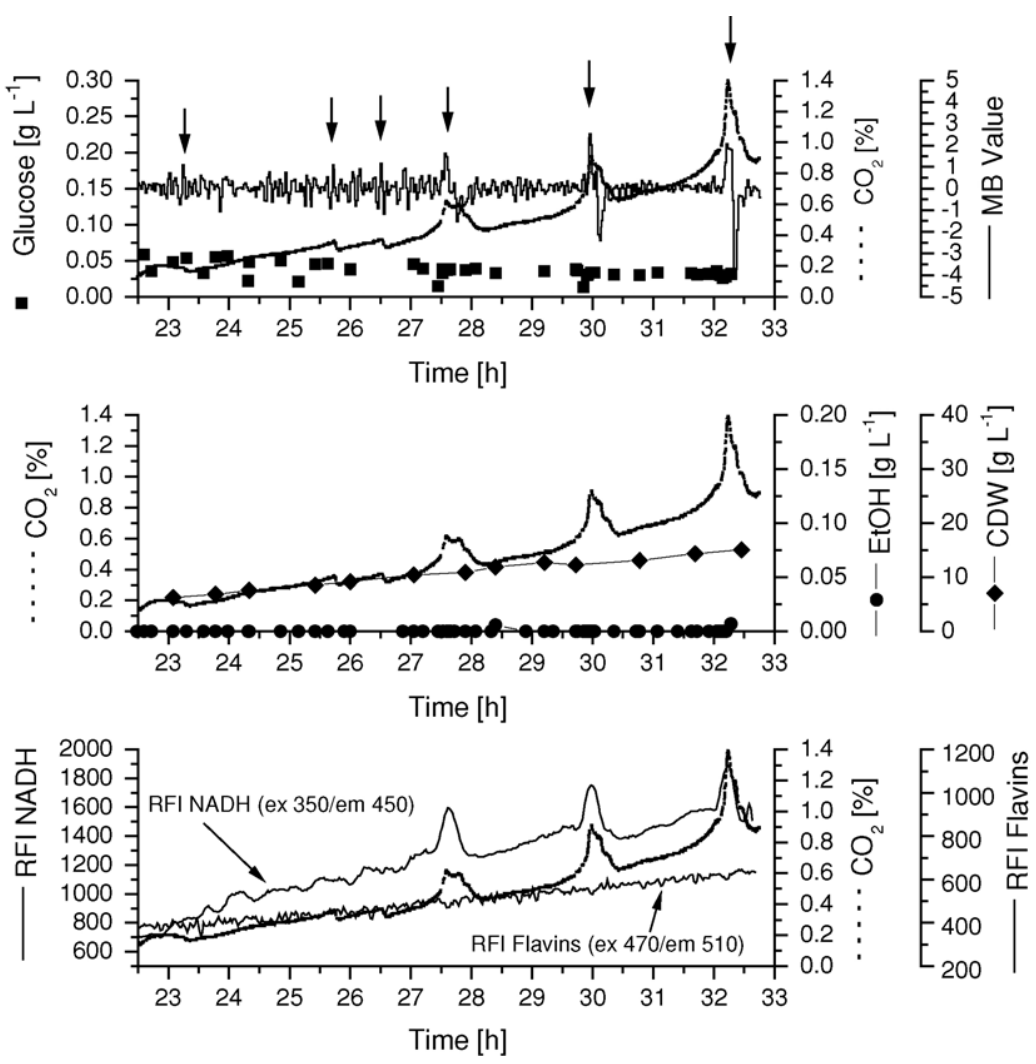

Fig. 4. S. cerevisiae fed-batch cultivation with fluorescence based glucose control. Time course of the calculated MB values, the carbon dioxide production, glucose and ethanol concentration, cellular dry weight (CDW) and of the relative fluorescence intensities (RFI) of NADH and flavins.

\section{Conclusions}

Since the cultivation of microorganisms as suppliers of various bioproducts has become more and more important in modern biotechnology there is a constant demand for the optimization of bioprocess monitoring and control. Optical sensors appear very promising, for they offer the possibility of non-invasive, nondestructive and continuous on-line monitoring. Twodimensional fluorescence spectroscopy in particular allows for the instantaneous detection of multiple biogenic fluorophors directly correlating to the metabolic state of the cells.

The presented approach for controlling S. cerevisiae fed-batch cultivations has proven to provide for an optimal glucose feed. Continuously recorded 2D fluorescence spectra served for the calculation of a metabolic value, which offers information about the present metabolic state and can be used to control the feed rate to prevent ethanol production. In comparison to most available optical sensor systems the BioView ${ }^{\circledR}$ sensor used for the fluorescence measurement is suitable for industrial environments.

\section{References}

Arndt, M., Hitzmann, B., 2004. Kalman filter based glucose control at small set points during fed-batch cultivation of Saccharomyces cerevisiae. Biotechnol. Prog. 20, 377-383.

Chen, C.W., Lei, B.C., Yeh, K.W., Duan, K.J., 2003. Recombinant sweet potato sporamin production via glucose/ $\mathrm{pH}$ control in fedbatch cultures of Saccharomyces cerevisiae. Proc. Biochem. 38 (8), 1223-1229.

Claes, J.E., Van Impe, J.F., 2000. Combining yield coefficients and exit-gas analysis for monitoring of the baker's yeast fed-batch fermentation. Bioproc. Eng. 22, 195-200.

Dairaku, K., Yamasaki, Y., Morikawa, H., Shioya, S., Takamatsu, T., 1982. Experimental study of time-optimal control in fed-batch culture of baker's yeast. J. Ferment. Technol. 60 (1), 67-75. 
De Dekken, R.H., 1966. The Crabtree effect: a regulatory system in yeast. J. Gen. Microbiol. 44, 149-156.

Gomy, C., Jouan, M., Dao, N.Q., 1988. A quantitative laser Raman spectrometric method with fibre optics for monitoring an alcoholic fermentation. Anal. Chim. Acta 215, 211-221.

Hitzmann, B., Broxtermann, O., Cha, Y.L., Sobieh, O., Stärk, E., Scheper, T., 2000. The control of glucose concentration during yeast fed-batch cultivation using a fast measurement complemented by an extended Kalman filter. Bioproc. Eng. 23, 337-341.

Jobé, A.M., Herwig, C., Surzyn, M., Walker, B., Marison, I., von Stockar, U., 2003. Generally applicable fed-batch culture concept based on the detection of metabolic state by on-line balancing. Biotechnol. Bioeng. 82 (6), 627-639.

Marose, S., Lindemann, C., Scheper, T., 1998. Two-dimensional fluorescence spectroscopy: a new tool for on-line bioprocess monitoring. Biotechnol. Prog. 14, 63-74.

Mazarevica, G., Diewok, J., Baena, J.R., Rosenberg, E., Lendl, B., 2004. On-line fermentation monitoring by mid-infrared spectroscopy. Appl. Spec. 58 (7), 804-810.

Pham, H.T., Larsson, G., Enfors, S.O., 1998. Growth and energy metabolism in aerobic fed-batch cultures of Saccharomyces cerevisiae: simulation and model verification. Biotechnol. Bioeng. 60 (4), 474-482.

Schatzmann, H., 1975. Anaerobes Wachstum von Saccharomyces cerevisiae. Dissertation, ETH Zürich No. 5504.

Scheper, T., Hitzmann, B., Stärk, E., Ulber, R., Faurie, R., Sosnitza, P., Reardon, K.F., 1999. Bioanalytics: detailed insight into bioprocesses. Anal. Chim. Acta 400, 121-143.

Schügerl, K., Bellgardt, K.H., Kretzmer, G., Hitzmann, B., Scheper, T., 1993. In-situ-und on-line-Überwachung und Regelung biotechnologischer Prozesse. CIT 65 (12), 1447-1456.
Shaw, A.D., Kaderbhai, N., Jones, A., Woodward, A.M., Goodacre, R., Rowland, J.J., Kell, D.B., 1999. Noninvasive, on-line monitoring of the biotransformation by yeast of glucose to ethanol using dispersive Raman spectroscopy and chemometrics. Appl. Spec. 53 (11), 1419-1428.

Shimizu, K., Morikawa, M., Mizutani, S., Iijima, S., Matsubara, M., Kobayashi, T., 1988. Comparison of control techniques for baker's yeast culture using an automatic glucose analyzer. J. Chem. Eng. Japan 21 (2), 113-117.

Sonnleitner, B., Käppeli, O., 1986. Growth of Saccharomyces cerevisiae is controlled by its limited respiratory capacity: formulation and verification of a hypothesis. Biotechnol. Bioeng. 28, 927-937.

Stärk, E., Hitzmann, B., Schügerl, K., Scheper, T., Fuchs, C., Köster, D., Märkl, H., 2002. In-situ-fluorescence-probes: a useful tool for non-invasive bioprocess monitoring. Adv. Biochem. Eng./Biotechnol. 74, 21-37.

Ulber, R., Frerichs, J.G., Beutel, S., 2003. Optical sensors for bioprocess monitoring. Anal. Bioanal. Chem. 376, 342 348.

Valentinotti, S., Srinivasan, B., Holmberg, U., Bonvin, D., Cannizaro, C., Rhiel, M., von Stockar, U., 2003. Optimal operation of fedbatch fermentations via adaptive control of overflow metabolite. Contr. Eng. Prac. 11 (6), 665-674.

Van Hoek, P., Van Dijken, J.P., Pronk, J.T., 1999. Effect of specific growth rate on fermentative capacity of baker's yeast. Appl. Environ. Microbiol. 64 (11), 4233-4266.

Woehrer, W., Roehr, M., 1981. Regulatory aspects of baker's yeast metabolism in aerobic fed-batch cultures. Biotechnol. Bioeng. $23,567-581$. 